\title{
NEUTRINO PAIR EMISSION FROM THERMALLY EXCITED NUCLEI IN STELLAR COLLAPSE
}

\author{
Alan A. Dzhioev ${ }^{1, *}$ and A. I. Vdovin ${ }^{1, * *}$ \\ ${ }^{1}$ Bogoliubov Laboratory of Theoretical Physics, JINR, 141980, Dubna, Russia. \\ We examine the rate of neutrino-antineutrino pair emission by hot nuclei in collaps- \\ ing stellar cores. The rates are calculated assuming that only allowed charge-neutral \\ Gamow-Teller $\left(\mathrm{GT}_{0}\right)$ transitions contribute to the decay of thermally excited nuclear \\ states. To obtain the $\mathrm{GT}_{0}$ transition matrix elements, we employ the quasiparticle \\ random phase approximation extended to finite temperatures within the thermo field \\ dynamics formalism. The decay rates and the energy emission rates are calculated for \\ the sample nuclei ${ }^{56} \mathrm{Fe}$ and ${ }^{82} \mathrm{Ge}$ at temperatures relevant to core collapse supernovae.
}

PACS: 26.50.+x; 23.40.-s; 21.60.Jz; 24.10Pa

\section{INTRODUCTION}

The significant role played by processes with neutrinos in core-collapse supernova is well known [1-3]. Until the core reaches the densities $\rho \gtrsim 10^{11} \mathrm{~g} \cdot \mathrm{cm}^{-3}$, almost all of the energy of the collapse is radiated by neutrinos that move out of the star freely. Energy emission from the core via neutrinos helps to maintain the low entropy and, as a result, nucleons reside primarily in nuclei at the nuclear matter density. At densities exceeding a few units of $10^{11} \mathrm{~g} \cdot \mathrm{cm}^{-3}$ high-energy neutrinos become trapped inside the core due to elastic scattering off nuclei. Due to this, the lepton fraction inside the core increases and favors a stronger shock wave responsible for the supernova explosion. However, low-energy neutrinos $E_{\nu}<10$ have the longer mean free path and can therefore diffuse more easily out of the core. There are a number of processes contributing to the low-energy neutrino production: the inelastic scattering of neutrinos on electrons [4], the neutrino-nucleus inelastic scattering [1], etc. To reveal the net effect of neutrinos on the core-collapse process all relevant neutrino reactions

\footnotetext{
* Electronic address: dzhioev@theor.jinr.ru

** Electronic address: vdovin@theor.jinr.ru
} 
should be included into the supernova neutrino transport calculations.

In this paper, we examine one of the possible sources of low-energy neutrinos, namely, the process of the neutrino - antineutrino $(\nu \bar{\nu})$ pair emission by an excited nucleus

$$
(A, Z)^{*} \rightarrow(A, Z)+\nu_{k}+\bar{\nu}_{k} .
$$

Here, the index $k=e, \mu, \tau$ corresponds to three neutrino flavors. The astrophysical importance of this process under the extreme condition of a stellar collapse (i.e., high temperatures and matter densities) was first recognized by Pontecorvo [5], who noticed that the neutrinopair emission by thermally excited nuclei is a powerful mechanism for the energy loss by stars. It was later pointed out by Bahcall et al. [6] that despite the low rate this process may be important because the thermal population of excited nuclear states can be substantial at stellar temperatures.

Consider an ensemble of nuclei in equilibrium with a thermal reservoir at temperature $T$. The role of such a reservoir is played by the stellar interior and the temperature is in the neighborhood of $0.5 \mathrm{MeV}$ to $2 \mathrm{MeV}\left(0.86 \mathrm{MeV} \approx 10^{10} \mathrm{~K}=10 \mathrm{GK}\right)$. Nuclear excited states are thermally populated according to the Boltzmann distribution $g_{i}=\left(2 J_{i}+1\right) \exp \left(-E_{i} / T\right)$, where $J_{i}$ and $E_{i}$ are the spin and the excitation energy of the level $i$. We are interested in the total decay rate from thermally excited nuclear states via the neutrino-pair emission as well as in the corresponding energy emission rate. In the nonrelativistic approximation only the Gamow-Teller (GT) down-transitions contribute to the decay rate $\lambda_{i f}$ from the nuclear state $i$ to the final nuclear state $f$ with the lower excitation energy $E_{f}[2,6,7]$

$$
\begin{aligned}
\lambda_{i f} & =3 \frac{G_{F}^{2} g_{A}^{2}}{60 \pi^{3} \hbar^{7} c^{6}}\left(\Delta E_{i f}\right)^{5} B\left(\mathrm{GT}_{0}\right)_{i f} \\
& =3 \lambda_{0}\left(\Delta E_{i f}\right)^{5} B\left(\mathrm{GT}_{0}\right)_{i f}, \quad \lambda_{0} \approx 1.72 \times 10^{-4} \mathrm{~s}^{-1} \mathrm{MeV}^{-5} .
\end{aligned}
$$

Here, $\Delta E_{i f}=E_{i}-E_{f}$ is the transition energy, $G_{F}$ is the Fermi weak coupling constant, $g_{A} \approx 1.26$, and $B\left(\mathrm{GT}_{0}\right)_{i f}$ is the reduced transition probability (strength) associated with the charge-neutral $\mathrm{GT}_{0}$ operator

$$
B\left(\mathrm{GT}_{0}\right)_{i f}=\left(2 J_{i}+1\right)^{-1}\left|\left\langle i\left\|\vec{\sigma} t_{0}\right\| f\right\rangle\right|^{2}
$$

where $\vec{\sigma}$ is the spin operator and $t_{0}$ is the zero component of the isospin operator. The factor of 3 in Eq. (2) takes into account three neutrino flavors. Knowing the partial decay rates 
$\lambda_{i f}$, the total decay rate $(\Lambda)$ and the energy emission rate $(P)$ can be evaluated by summing over the Boltzmann-weighted, individually determined contributions

$$
\Lambda=Z^{-1}(T) \sum_{i, f} g_{i} \lambda_{i f}
$$

and

$$
P=Z^{-1}(T) \sum_{i, f} g_{i} \Delta E_{i f} \lambda_{i f}
$$

where $Z=\sum_{i} g_{i}$ is the partition function.

In the pioneering work by Bahcall et al. [6] the neutrino-pair emission was considered at relatively low temperatures when only a few low-lying nuclear states contribute significantly to the sum $P(5)$. The corresponding Gamow-Teller transition matrix elements between nuclear excited and ground states were obtained from the experimental decay rate for the $M 1 \gamma$-emission. At higher temperatures an explicit state-by-state evaluation of the sums in Eqs. (4)-(5) is not feasible because transitions between nuclear states at high excitations contribute to the sum significantly ${ }^{1}$. For such transitions the nuclear level properties and hence the Gamow-Teller matrix elements are generally unknown. In the work by Crawford et al. [7], the neutrino energy emission rate for individual nuclei was parameterized for temperatures in the range $0.08 \leq T_{10} \leq 0.6\left(T_{10}=T / 10^{10} \mathrm{~K}\right)$ by replacing the discrete energy levels with the statistical energy level density when considering the $\mathrm{GT}_{0}$ transitions from a highly excited state. The $\mathrm{GT}_{0}$ matrix elements were estimated using the method similar to that in [6]. Later on, the neutrino-pair emission at high temperatures was considered by Kolb and Mazurek [8] making use of the strength function obtained within the Fermi-gas approach. These calculations were improved by Fuller and Meyer [2] using the independent single-particle shell model.

In the present paper, we apply the alternative approach to study the neutrino-pair emission from thermally excited nuclei. Our approach is based on the thermal quasiparticle random phase approximation (TQRPA). We apply it in the context of thermo field dynamics (TFD) [9-11] which enables a transparent treatment of excitation and de-excitation processes and offers the possibility for systematic improvements. This approach was recently

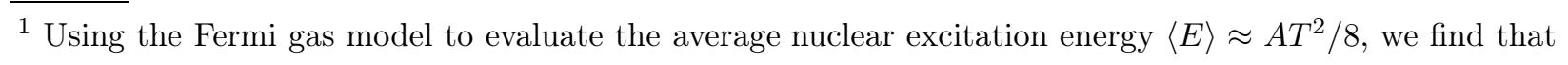
$\langle E\rangle \sim 30 \mathrm{MeV}$ for the iron-group nuclei $(A \approx 50-60)$ and for temperatures $T \sim 2 \mathrm{MeV}$. 
used in studies of the electron capture and neutrino inelastic scattering on hot nuclei under supernova conditions $[12,13]$.

\section{FORMALISM}

The details of our approach are expounded in $[12,13]$. Here, we briefly outline the key points relevant for the present discussion. In TFD, a formal doubling of the degrees of freedom of a nucleus (in the present case) is introduced: a tilde conjugate operator $\widetilde{A}$ (acting in the independent "tilde" Hilbert space) is associated with an ordinary operator $A$ (acting in the original Hilbert space) in accordance with properly formulated tilde conjugation rules [9$11]^{2}$. The essential ingredients of TFD are the thermal vacuum $|0(T)\rangle$ and the thermal Hamiltonian $\mathcal{H}$. The thermal vacuum describes equilibrium properties of the system. For instance, the grand canonical average of any operator can be calculated as the expectation value with respect to $|0(T)\rangle$. Excited states of the system at finite temperature correspond to eigenstates of the thermal Hamiltonian. The latter is defined as the difference between the original system Hamiltonian $H$ and its tilde counterpart $\widetilde{H}, \mathcal{H}=H-\widetilde{H}$. The thermal vacuum is the zero-energy eigenstate of the thermal Hamiltonian $\mathcal{H}$ satisfying the thermal state condition $[9-11]$

$$
A|0(T)\rangle=\sigma \mathrm{e}^{\mathcal{H} / 2 T} \widetilde{A}^{\dagger}|0(T)\rangle
$$

where $\sigma=1$ for bosonic operators $A$ and $\sigma=i$ for fermionic operators.

As it follows from the definition of $\mathcal{H}$, each of its eigenstates with positive energy has its counterpart - the tilde-conjugate eigenstate - with negative but the same absolute energy value. Transitions from the thermal vacuum to positive energy states (up-transitions) correspond to excitation of the system, while transitions to negative energy states (downtransitions) describe decay of thermally excited states. The transition probabilities are given by matrix elements of a transition operator between the thermal vacuum and the excited state.

In the most practical cases one cannot diagonalize $\mathcal{H}$ exactly. Let us assume that we diagonalize the thermal nuclear Hamiltonian by invoking some approximation methods (e.g.,

\footnotetext{
2 The correspondence between the thermo field dynamics and the superoperator formalism used one of the authors (A.D.) to study nonequilibrium electron transport phenomena (see, e.g., [14]) is discussed in [15].
} 
the mean-field or random phase approximations $[16,17])$. Then, the thermal Hamiltonian can be written as

$$
\mathcal{H} \approx \sum_{i} \omega_{i}(T)\left(Q_{i}^{\dagger} Q_{i}-\widetilde{Q}_{i}^{\dagger} \widetilde{Q}_{i}\right)
$$

where $\omega_{i}(T)>0$. The approximate thermal vacuum, $|0(T)\rangle$, is the vacuum state for $Q_{i}, \widetilde{Q}_{i}$ operators. It obeys the thermal state condition (6), where instead of the exact thermal Hamiltonian we use $\mathcal{H}$ given by (7).

Applying the above formalism to the problem of the neutrino-pair emission by a hot nucleus we find that the partial decay rate from the thermal vacuum to the tilde state $\widetilde{Q}_{i}$ is given by

$$
\lambda_{i}=3 \lambda_{0} \omega_{i}^{5} \widetilde{\Phi}_{i}
$$

Here, $\omega_{i}$ is the transition energy (i.e. energy released per decay) and

$$
\widetilde{\Phi}_{i}=\left|\left\langle\widetilde{Q}_{i}\left\|\vec{\sigma} t_{0}\right\| 0(T)\right\rangle\right|^{2}
$$

is the $\mathrm{GT}_{0}$ strength for a down-transition. Note, that the $\mathrm{GT}_{0}$ strength for up-transitions, $\Phi_{i}$, can be obtained from (9) with the help of the detailed balance principle

$$
\Phi_{i}=\left|\left\langle Q_{i}\left\|\vec{\sigma} t_{0}\right\| 0(T)\right\rangle\right|^{2}=\exp \left(\omega_{i} / T\right) \widetilde{\Phi}_{i}
$$

The partial decay rate (8) determines the spectrum of emitted neutrino-antineutrino pairs. Summing over transition energy gives the total decay rate

$$
\Lambda=\sum_{i} \lambda_{i}
$$

and the energy emission rate

$$
P=\sum_{i} \omega_{i} \lambda_{i}
$$

To obtain the $\mathrm{GT}_{0}$ strength distribution for a hot nucleus we employ the Hamiltonian of the Quasiparticle-Phonon Model (QPM) $H_{\mathrm{QPM}}$ [18]. The QPM Hamiltonian consists of the proton and neutron mean fields (the part $H_{\mathrm{sp}}$ ), the BCS pairing interaction $H_{\text {pair }}$, and the separable isoscalar and isovector particle-hole interaction $H_{\mathrm{ph}}$

$$
H_{\mathrm{QPM}}=H_{\mathrm{sp}}+H_{\mathrm{pair}}+H_{\mathrm{ph}}
$$


The three terms of $H_{\mathrm{QPM}}$ read

$$
\begin{aligned}
H_{\mathrm{sp}} & =\sum_{\tau=p, n} \sum_{j m}^{\tau}\left(E_{j}-\lambda_{\tau}\right) a_{j m}^{\dagger} a_{j m}, \\
H_{\mathrm{pair}} & =-\frac{1}{4} \sum_{\tau=p, n} G_{\tau} \sum_{\substack{j m \\
j^{\prime} m^{\prime}}}^{\tau} a_{j m}^{\dagger} a_{\frac{\jmath m}{\dagger}}^{\dagger} a_{\jmath^{\prime} m^{\prime}} a_{j^{\prime} m^{\prime}}, \\
H_{\mathrm{ph}} & =-\frac{1}{2} \sum_{L \lambda}\left(\kappa_{0}^{(L \lambda)}+\kappa_{1}^{(L \lambda)} \vec{\tau}_{1} \vec{\tau}_{2}\right) \sum_{\mu} S_{L \lambda \mu}^{\dagger} S_{L \lambda \mu},
\end{aligned}
$$

where

$$
S_{L \lambda \mu}^{\dagger}=\sum_{\tau=p, n} \sum_{\substack{j m \\ j^{\prime} m^{\prime}}}^{\tau}\left\langle j m\left|i^{L} r^{L}\left[Y_{L} \vec{\sigma}\right]_{\mu}^{\lambda}\right| j^{\prime} m^{\prime}\right\rangle a_{j m}^{\dagger} a_{j^{\prime} m^{\prime}} .
$$

The quantum numbers $j, m$ actually represent the complete set of single-particle quantum numbers $n, l, j, m, \tau\left(\tau=n, p\right.$ is the isotopic index) and $a_{\overline{\jmath m}}=(-1)^{j-m} a_{j-m}$. The notation $\sum^{\tau}$ implies the summation over neutron $(\tau=n)$ or proton $(\tau=p)$ single-particle states only. Since we assume that only $\mathrm{GT}_{0}\left(J^{\pi}=1^{+}\right)$transitions contribute to the neutrino-pair emission, only the spin-multipole part of the particle-hole interaction is taken into account (actually, we need only the spin-multipole operators with $\lambda=1, L=0,2$ ). The parameters $\kappa_{0}^{(L \lambda)}$ and $\kappa_{1}^{(L \lambda)}$ are the strength parameters of the isoscalar and isovector spin-multipole forces, respectively.

To determine the thermal behavior of a nucleus governed by the Hamiltonian (13), we should diagonalize the thermal Hamiltonian $\mathcal{H}_{\mathrm{QPM}}=H_{\mathrm{QPM}}-\widetilde{H}_{\mathrm{QPM}}$ and find the corresponding thermal vacuum state. Within TQRPA, this can be done in two steps.

As the first step, the sum of the single-particle and pairing terms $\mathcal{H}_{\mathrm{BCS}}=\mathcal{H}_{\mathrm{sp}}+\mathcal{H}_{\text {pair }}$ is diagonalized. To this end, two subsequent unitary transformations are made. The first is the usual Bogoliubov $u, v$ transformation from the original particle operators $a_{j m}^{\dagger}, a_{j m}$ to the Bogoliubov quasiparticle ones $\alpha_{j m}^{\dagger}, \alpha_{j m}$. The same transformation is applied to the tilde operators $\widetilde{a}_{j m}^{\dagger}, \widetilde{a}_{j m}$, thus producing the tilde quasiparticle operators $\widetilde{\alpha}_{j m}^{\dagger}, \widetilde{\alpha}_{j m}$. The second, unitary thermal Bogoliubov transformation, mixes non-tilde and tilde quasiparticles and introduces thermal quasiparticles

$$
\begin{aligned}
& \beta_{j m}^{\dagger}=x_{j} \alpha_{j m}^{\dagger}-i y_{j} \widetilde{\alpha}_{j m} \\
& \widetilde{\beta}_{j m}^{\dagger}=x_{j} \widetilde{\alpha}_{j m}^{\dagger}+i y_{j} \alpha_{j m} \quad\left(x_{j}^{2}+y^{2}=1\right) .
\end{aligned}
$$

The coefficients of both transformations are found by diagonalizing the single-particle part 
of $\mathcal{H}_{\mathrm{BCS}}$, i.e.

$$
\mathcal{H}_{\mathrm{BCS}} \simeq \sum_{\tau} \sum_{j m}^{\tau} \varepsilon_{j}(T)\left(\beta_{j m}^{\dagger} \beta_{j m}-\widetilde{\beta}_{j m}^{\dagger} \widetilde{\beta}_{j m}\right),
$$

and demanding that the vacuum of thermal quasiparticles, $|0(T) ; \mathrm{qp}\rangle$, obeys the thermal state condition (6) with the Hamiltonian (15). As a result we obtain the well-known equation of the finite-temperature BCS. The coefficients $y_{j}^{2}$ determine the average number of thermally excited Bogoliubov quasiparticles and coincide with thermal occupation factors of the Fermi-Dirac statistics. Because of thermally excited Bogoliubov quasiparticles, singleparticle transitions which are blocked at zero temperature become thermally unblocked at finite temperatures. In particular, these are single-particle down-transitions from high-lying levels to low-lying ones.

As the second step of the approximate diagonalization of $\mathcal{H}_{\mathrm{QPM}}$, the particle-hole correlations are taken into account within the thermal QRPA (TQRPA). To this end, we diagonalize $\mathcal{H}_{\mathrm{QPM}}$ in terms of thermal phonon operators

$$
\mathcal{H}_{\mathrm{TRPA}}=\sum_{\lambda \mu i} \omega_{\lambda i}(T)\left(Q_{\lambda \mu i}^{\dagger} Q_{\lambda \mu i}-\widetilde{Q}_{\lambda \mu i}^{\dagger} \widetilde{Q}_{\lambda \mu i}\right)
$$

where $\omega_{\lambda i}(T)>0$. The thermal phonon operator is defined as a linear superposition of various pairs (6 variants in total) of the creation and annihilation ordinary- and tilde thermal quasiparticle operators

$$
\begin{aligned}
Q_{\lambda \mu i}^{\dagger}=\frac{1}{2} \sum_{\tau} \sum_{j_{1} j_{2}}^{\tau}\left\{\psi_{j_{1} j_{2}}^{\lambda i}\left[\beta_{j_{1}}^{\dagger} \beta_{j_{2}}^{\dagger}\right]_{\mu}^{\lambda}\right. & +\widetilde{\psi}_{j_{1} j_{2}}^{\lambda i}\left[\widetilde{\beta}_{\overline{J_{1}}}^{\dagger} \widetilde{\beta}_{\overline{j_{2}}}^{\dagger}\right]_{\mu}^{\lambda}+2 i \eta_{j_{1} j_{2}}^{\lambda i}\left[\beta_{j_{1}}^{\dagger} \widetilde{\beta}_{\bar{\jmath}_{2}}^{\dagger}\right]_{\mu}^{\lambda} \\
& \left.+\phi_{j_{1} j_{2}}^{\lambda i}\left[\beta \overline{\overline{J_{1}}} \beta_{\overline{\bar{J}_{2}}}\right]_{\mu}^{\lambda}+\widetilde{\phi}_{j_{1} j_{2}}^{\lambda i}\left[\widetilde{\beta}_{j_{1}} \widetilde{\beta}_{j_{2}}\right]_{\mu}^{\lambda}+2 i \xi_{j_{1} j_{2}}^{\lambda i}\left[\beta_{\overline{J_{1}}} \widetilde{\beta}_{j_{2}}\right]_{\mu}^{\lambda}\right\}
\end{aligned}
$$

where []$_{\mu}^{\lambda}$ denotes the coupling of single-particle angular momenta $j_{1}, j_{2}$ to the total angular momentum $\lambda$. The tilde phonon operator $\widetilde{Q}_{\lambda \mu i}^{\dagger}$ can be obtained from (17) by applying the tilde conjugation rules. The thermal phonon operators are considered as bosonic ones, which imposes the normalization constraint on the phonon amplitudes. To find the energy and the structure of thermal phonons, we apply the variational principle under the additional constraint that the vacuum of thermal phonons obeys the thermal state condition (6). As a result, we obtain the system of linear equations for the amplitudes $\psi_{j_{1} j_{2}}^{\lambda i}, \widetilde{\psi}_{j_{1} j_{2}}^{\lambda i}, \eta_{j_{1} j_{2}}^{\lambda i}$, etc. The solvability condition for this system yields the spectrum of thermal phonons (details can be found in Ref. [17]). These constitute the equations for the thermal quasiparticle random 
phase approximation. The vacuum of thermal phonons, $|0(T) ; \mathrm{ph}\rangle$ is the thermal vacuum in TQRPA.

Once the structure of thermal phonons is determined, one can evaluate the transition probabilities from the thermal vacuum to thermal one-phonon states. As was mentioned above, to describe decay processes, we need to take into account down-transitions from the thermal vacuum to tilde states. Considering the Gamow-Teller operator $\vec{\sigma} t_{0}$ we find the explicit expression for the transition probability (9) in TQRPA (below $\lambda=1$ )

$$
\begin{aligned}
\widetilde{\Phi}_{i}= & \frac{1}{4}\left[\sum_{\tau} \sum_{j_{1} j_{2}}^{\tau}\left\langle j_{1}\left\|\vec{\sigma} t_{0}\right\| j_{2}\right\rangle \times\right. \\
& \left.\times\left\{u_{j_{1} j_{2}}^{(-)}\left(x_{j_{1}} x_{j_{2}} \widetilde{w}_{j_{1} j_{2}}^{\lambda i}-y_{j_{1}} y_{j_{2}} w_{j_{1} j_{2}}^{\lambda i}\right)+v_{j_{1} j_{2}}^{(+)}\left(x_{j_{1}} y_{j_{2}} \widetilde{s}_{j_{1} j_{2}}^{\lambda i}-y_{j_{1}} x_{j_{2}} s_{j_{1} j_{2}}^{\lambda i}\right)\right\}\right]^{2} .
\end{aligned}
$$

Here, $\left\langle j_{1}\left\|\vec{\sigma} t_{0}\right\| j_{2}\right\rangle$ is the reduced single-particle matrix element of the $\mathrm{GT}_{0}$ operator; $u_{j_{1} j_{2}}^{(-)}=$ $u_{j_{1}} v_{j_{2}}-v_{j_{1}} u_{j_{2}}, v_{j_{1} j_{2}}^{(+)}=u_{j_{1}} u_{j_{2}}+v_{j_{1}} v_{j_{2}}$; and $w_{j_{1} j_{2}}^{\lambda i}=\psi_{j_{1} j_{2}}^{\lambda i}-\phi_{j_{1} j_{2}}^{\lambda i}, s_{j_{1} j_{2}}^{\lambda i}=\eta_{j_{1} j_{2}}^{\lambda i}-\xi_{j_{1} j_{2}}^{\lambda i}$, etc. Substituting $\omega_{\lambda i}$ and $\widetilde{\Phi}_{i}$ into (8) we find the partial decay rates from the TQRPA thermal vacuum to the tilde one-phonon states. Then, we can find the total decay rate Eq. (11) and the energy emission rate Eq. (12).

\section{CALCULATIONS FOR THE HOT NUCLEUS ${ }^{56} \mathrm{FE}$ AND ${ }^{82} \mathrm{GE}$}

Numerical calculations of neutrino-pair emission rates have been performed for the iron isotope ${ }^{56} \mathrm{Fe}$ and the neutron-rich germanium isotope ${ }^{82} \mathrm{Ge}$. The iron isotope is one of the most astrophysically relevant nuclei for the early presupernova collapse. Neutron-rich nuclei dominate the nuclear composition at a later stage of collapse. For the considered nuclei the single-particle wave functions and energies are calculated with the spherically symmetric Woods-Saxon potential with the parameters from [19]. For ${ }^{82}$ Ge the sequence of singleparticle levels appears to be close to that from Ref. [20]. Within the independent singleparticle shell-model ${ }^{82} \mathrm{Ge}$ has all the neutron $p f$-shell orbits filled with the valence neutrons in the $s d g$ shell and the valence protons in the $p f$-shell.

The constants of the pairing interaction, $G_{p, n}$, are determined to reproduce experimental pair energies in the BCS approximation. The pairing does not affect the obtained results at temperatures above the critical one, $T_{\mathrm{cr}} \approx 0.8 \mathrm{MeV}$, when pairing correlations vanish.

As it was pointed out in the previous section, $J^{\pi}=1^{+}$excitations in spherically symmetric nuclei are generated by the spin-multipole part of the particle-hole interaction or, more 
precisely, by its spin-monopole and spin-quadrupole components. To determine the isovector, $\kappa_{1}^{01}, \kappa_{1}^{21}$, and isoscalar, $\kappa_{0}^{01}, \kappa_{0}^{21}$, coupling parameters, we use the standard estimates for the separable schematic forces with radial form factors $r^{\lambda}$ [21].

First, we have performed TQRPA calculations of the $\mathrm{GT}_{0}$ strength distribution in ${ }^{56} \mathrm{Fe}$ and ${ }^{82}$ Ge. In Fig. 1, we display at three different temperatures the strength distributions of $\mathrm{GT}_{0}$ transitions from the thermal vacuum to tilde one-phonon states. We recall that only these down-transitions contribute to the decay process. All figures are plotted as a function of the transition energy $\omega_{i}>0$. While the details of the strength distributions vary between two nuclei, the essential features are the same. Namely, at low temperatures $(T=0.5 \mathrm{MeV})$ the transition strength is concentrated in a narrow resonance structure within the energy region between 2 and $3 \mathrm{MeV}$. With increasing temperature one observes the increase in the total $\mathrm{GT}_{0}$ strength accompanied by the appearance of higher energy transitions. Referring to Fig. 1, one sees that the high-energy $\mathrm{GT}_{0}$ transition strength is more fragmented than the low-energy one.

All these features can be readily explained. First of all, we note that only transitions between the levels which are the spin-orbit partners contribute to the $\mathrm{GT}_{0}$ strength. As a consequence, the transition energies are determined by the spin-orbit splitting in the nucleus under study. Some increase in the transition energy comes from the pairing effects and the repulsive particle-hole interaction.

At low temperatures, single-particle down-transitions between levels close to proton and neutron Fermi surfaces are effectively thermally unblocked. For ${ }^{56} \mathrm{Fe}$ these transitions are $2 p_{1 / 2} \rightarrow 2 p_{3 / 2}$ for both protons and neutrons. In the neutron-rich nucleus ${ }^{82} \mathrm{Ge}$ the neutron level $2 p_{3 / 2}$ is well below the Fermi level and, therefore, it is mostly filled at low temperatures. As a result, only the proton single-particle down-transition $2 p_{1 / 2} \rightarrow 2 p_{3 / 2}$ is thermally unblocked in ${ }^{82} \mathrm{Ge}$ at low $T$. Thus, at low temperatures the energy of thermally unblocked $\mathrm{GT}_{0}$ transitions is given by the spin-orbit splitting of the $2 p$ shell, which is in the range $2-3 \mathrm{MeV}$.

Increasing temperature promotes nucleons to high-lying single-particle levels. This makes possible down-transitions between spin-orbit partners with a large spin-orbit splitting. In ${ }^{56}$ Fe these transitions are $1 f_{7 / 2} \rightarrow 1 f_{5 / 2}$ and $1 g_{9 / 2} \rightarrow 1 g_{7 / 2}$ for both neutrons and protons. The fragmentation of the high-energy $\mathrm{GT}_{0}$ strength, which is most pronounced at high temperatures $T=2.5 \mathrm{MeV}$, reflects the distinction in the spin-orbit splitting of $1 f$ and $1 g$ 
shells. The same is true for ${ }^{82} \mathrm{Ge}$, with the only difference that the $1 h_{11 / 2} \rightarrow 1 h_{9 / 2}$ neutron transition contributes to the $\mathrm{GT}_{0}$ strength above $10 \mathrm{MeV}$.

According to Eq. (8), the transition strengths should be multiplied by the fifth power of the transition energy to give partial decay rates or, what is the same, the spectrum of emitted neutrino pairs. Figure 2 shows partial decay rates computed in this fashion for considered nuclei at temperatures $T=0.5,1.5$ and $2.5 \mathrm{MeV}$. As is obvious from the figure, multiplying by $\omega_{i}^{5}$ clearly favors high-energy transitions. The effect is most pronounced at intermediate temperatures. Comparing Figs. 1 and 2 , we see that at $T=1.5 \mathrm{MeV}$ the $\mathrm{GT}_{0}$ strength for down-transitions is mostly concentrated in the range $2-3 \mathrm{MeV}$, whereas the spectrum of the emitted neutrino-pair peaks at much higher energies. Thus, we conclude that the temperature growth increases the energy of emitted neutrinos. We will return to this point at the end of this Section.

In Fig. 3, the total decay rates $\Lambda$ (11) and energy emission rates $P(12)$ for ${ }^{56} \mathrm{Fe}$ and ${ }^{82} \mathrm{Ge}$ are shown as functions of $T$. As expected, both the rates demonstrate a strong temperature dependence. From the above discussion it becomes clear that the main reason for this is the thermal unblocking of high-energy $\mathrm{GT}_{0}$ down-transitions, since more strength at higher $\omega$ means faster emission rates. Moreover, we find that at the considered $T$ values the calculated rates in ${ }^{56} \mathrm{Fe}$ and ${ }^{82} \mathrm{Ge}$ are close to each other despite a considerable difference in their masses. This result is not surprising since in both the nuclei the same single-particle down-transitions dominate the neutrino-emission process. Comparing the obtained energy emission rate for ${ }^{56} \mathrm{Fe}$ with earlier estimates $[2,8]$ we find that our results are very close to those obtained in [2] by applying the independent single-particle shell-model.

Given the energy emission rates and the total decay rates we can find a mean energy of emitted neutrino pairs as $\langle E\rangle=P / \Lambda$. The results of these calculations are shown in Fig. 4. As evident from the figure, at low $T$ the mean energy of the emitted neutrino-pairs is less than $9 \mathrm{MeV}$ and $\langle E\rangle$ rises rapidly with temperature till $T \approx 1.0 \mathrm{MeV}$. After that $\langle E\rangle$ becomes nearly independent of $T$. Thus, for $T>1.0 \mathrm{MeV}$ the mean energy of the emitted neutrino-pairs is determined by the spin-orbit splitting of $f, g$ and $h$ shells. The last observation is important for neutron-rich nuclei with $A \sim 100$. Here, we would like to stress that for $T>2.0 \mathrm{MeV}$ the contribution of the fist-forbidden high-energy transitions is not negligible and should be taken into account (see Ref. [2]). By doing so we expect to observe an increase in $\langle E\rangle$ for $T>2.0 \mathrm{MeV}$. 


\section{CONCLUSIONS}

We have performed studies of a neutrino-antineutrino pair emission process from thermally excited nuclei. The studies are relevant for core collapse supernova simulations, as the considered process may play an important role in production of low-energy neutrinos and, hence, in the energy transport during the collapse. As an example, the neutrino-pair emission from ${ }^{56} \mathrm{Fe}$ and ${ }^{82} \mathrm{Ge}$ was considered for temperatures $0.5 \leq T \leq 2.5 \mathrm{MeV}$ by taking into account the allowed $\mathrm{GT}_{0}$ transitions only. Thermal effects were treated within the thermal quasiparticle random phase approximation in the context of the thermo field dynamics formalism.

Throughout the paper, we discussed the underlying nuclear physics of this process in terms of the single-particle down-transitions between the spin-orbit partner levels. It was found that the $f, g$ and $h$ shell spin-orbit transitions dominate the processes for $T \gtrsim 1 \mathrm{MeV}$, whereas the $p$ shell spin-orbit transitions are important only at lower $T$. Our calculations revealed the same effect as was found in $[2,8]$ : A temperature increase leads to a considerable enhance of the neutrino-pair emission rate. According to our calculations, this enhancement is due to the thermally unblocked $f, g$ and $h$ spin-orbit down-transitions. The last observation is important for neutron-rich nuclei.

The calculated energy emission rate for ${ }^{56} \mathrm{Fe}$ is close to that obtained in [2] within the independent single-particle shell-model. To extend our calculations to higher temperatures, we need to take into account the first-forbidden transitions. This will be the subject of our future studies.

1. S. W. Bruenn and W. C. Haxton, Astrophys. J. 376, 678 (1991).

2. G. M. Fuller and B. S. Meyer, Astrophys. J. 376, 701 (1991).

3. H.-Th. Janka et al., Phys. Rep. 442, 38 (2007).

4. D. L. Tubbs and D. N. Schramm, Astrophy. J. 201, 467 (1975).

5. B. Pontecorvo, Phys. Lett. 1, 287 (1962).

6. J. N. Bahcall et al., Phys. Lett. B 52, 275 (1974).

7. J. P. Crawford et al., Astrophys. J. 206, 208 (1976).

8. E. W. Kolb and T. J. Mazurek, Astrophys. J. 234, 1085 (1979). 
9. Y. Takahashi and H. Umezawa, Collective Phenomena 2, 55 (1975).

10. H. Umezawa, H. Matsumoto, M. Tachiki, Thermo field dynamics and condensed states (NorthHolland, Amsterdam, 1982).

11. I. Ojima, Ann. Phys. 137, 1 (1981).

12. A. A. Dzhioev et al., Phys. Rev. C 81, 015804 (2010).

13. A. A. Dzhioev et al., Phys. At. Nucl. 74, 1162 (2011).

14. A. A. Dzhioev and D. .S. Kosov, J. Phys.: Condens. Matter 24, 225304 (2012).

15. M. Schmutz, Z. Physik B 30, 97 (1978).

16. T. Hatsuda, Nucl. Phys. A 492, 187 (1989).

17. A. A. Dzhioev and A. I. Vdovin, Intern. J. Mod. Phys. E 18, 1535 (2009).

18. V. G. Soloviev, Theory of Atomic Nuclei: Quasiparticles and Phonons (Inst. Phys. Publ., Bristol, Philadelphia, 1992).

19. V. A. Chepurnov, Yad. Fiz. 6, 955 (1967).

20. J. Cooperstein and J. Wambach, Nucl. Phys. A 420, 591 (1984).

21. B. Castel and I. Hamamoto, Phys. Lett. B 65, 27 (1976). 


\section{Figure captions}

Fig.1 The Gamow-Teller strength distribution for down-transitions in ${ }^{56} \mathrm{Fe}$ (left panels) and ${ }^{82} \mathrm{Ge}$ (right panels) at three values of temperature: $T=0.5 \mathrm{MeV}$ (upper panels), $T=1.5 \mathrm{MeV}$ (middle panels), and $T=2.5 \mathrm{MeV}$ (lower panels). For each value of $T$ the total strength of down-transitions is displayed.

Fig.2 Partial decay rates (8) for ${ }^{56} \mathrm{Fe}$ (left panels) and ${ }^{82} \mathrm{Ge}$ (right panels) as functions of the transition energy. The partial decay rates are shown for three values of temperature: $T=0.5 \mathrm{MeV}$ (upper panels), $T=1.5 \mathrm{MeV}$ (middle panels) and $T=2.5 \mathrm{MeV}$ (lower panels).

Fig.3 The total decay rate $\Lambda$ (upper panel) and the energy emission rate $P$ (lower panel) for ${ }^{56} \mathrm{Fe}$ and ${ }^{82} \mathrm{Ge}$ as functions of $T$.

Fig.4 The mean energy $\langle E\rangle$ of $\nu \bar{\nu}$ pairs emitted from ${ }^{56} \mathrm{Fe}$ and ${ }^{82} \mathrm{Ge}$ as a function of temperature $T$. 

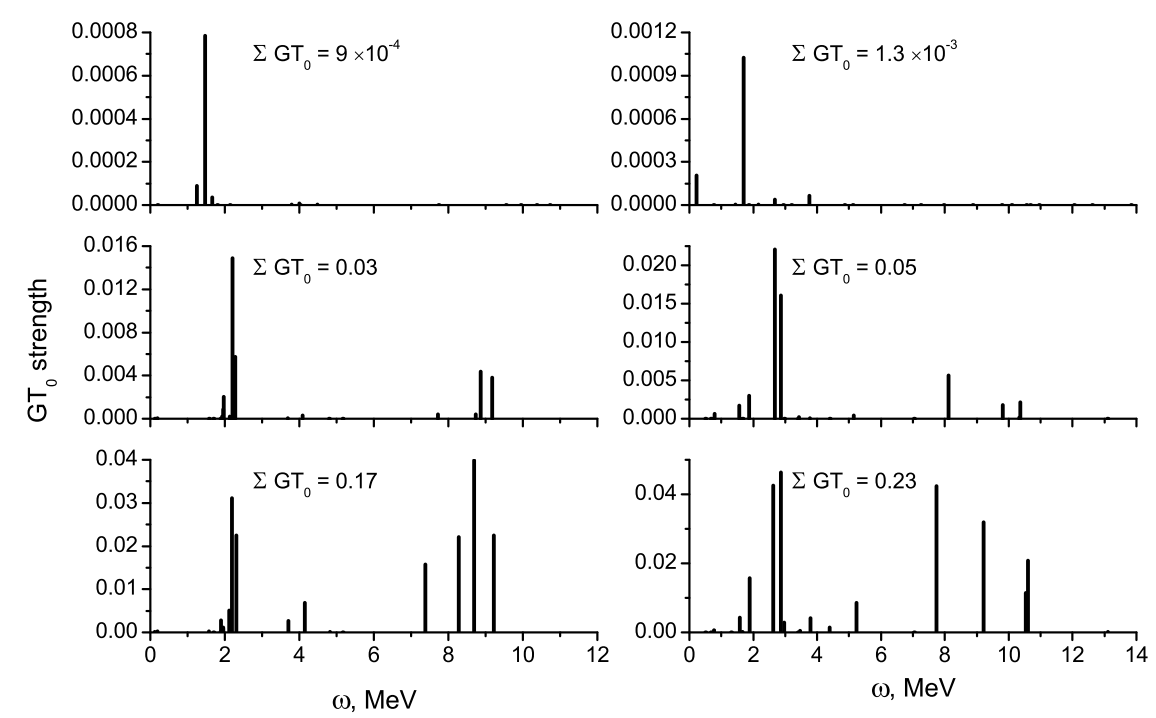

Figure 1. The Gamow-Teller strength distribution for down-transitions in ${ }^{56} \mathrm{Fe}$ (left panels) and ${ }^{82} \mathrm{Ge}$ (right panels) at three values of temperature: $T=0.5 \mathrm{MeV}$ (upper panels), $T=1.5 \mathrm{MeV}$ (middle panels), and $T=2.5 \mathrm{MeV}$ (lower panels). For each value of $T$ the total strength of down-transitions is displayed.
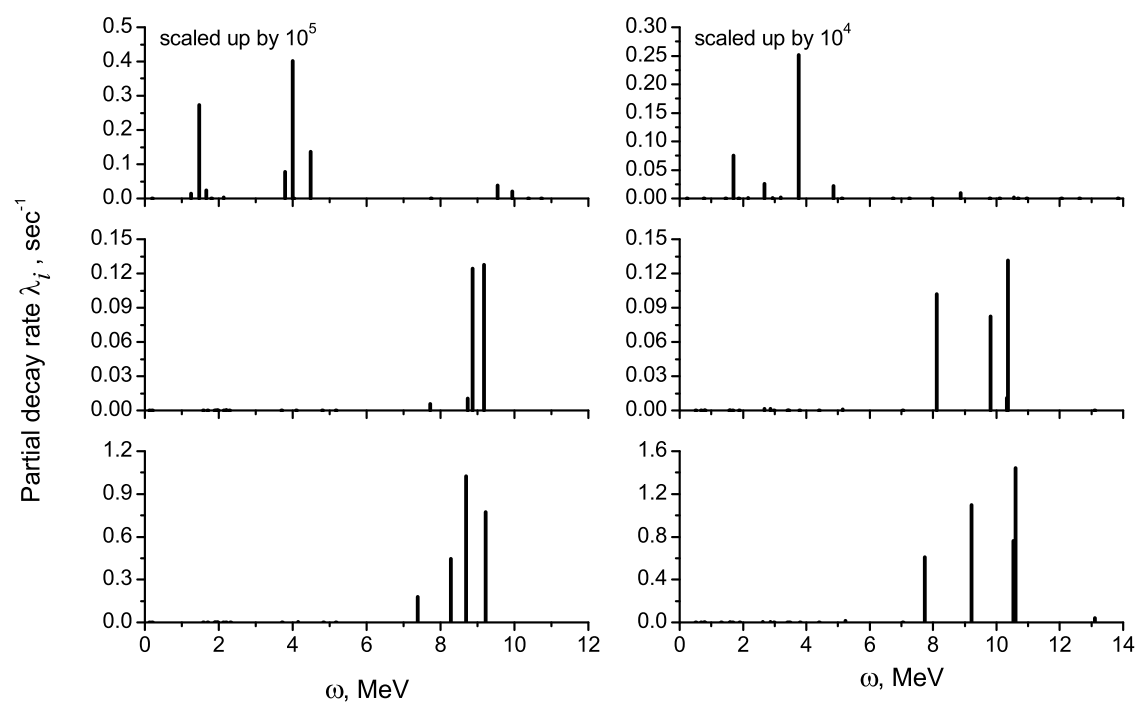

Figure 2. Partial decay rates (8) for ${ }^{56} \mathrm{Fe}$ (left panels) and ${ }^{82} \mathrm{Ge}$ (right panels) as functions of the transition energy. The partial decay rates are shown for three values of temperature: $T=0.5 \mathrm{MeV}$ (upper panels), $T=1.5 \mathrm{MeV}$ (middle panels) and $T=2.5 \mathrm{MeV}$ (lower panels). 

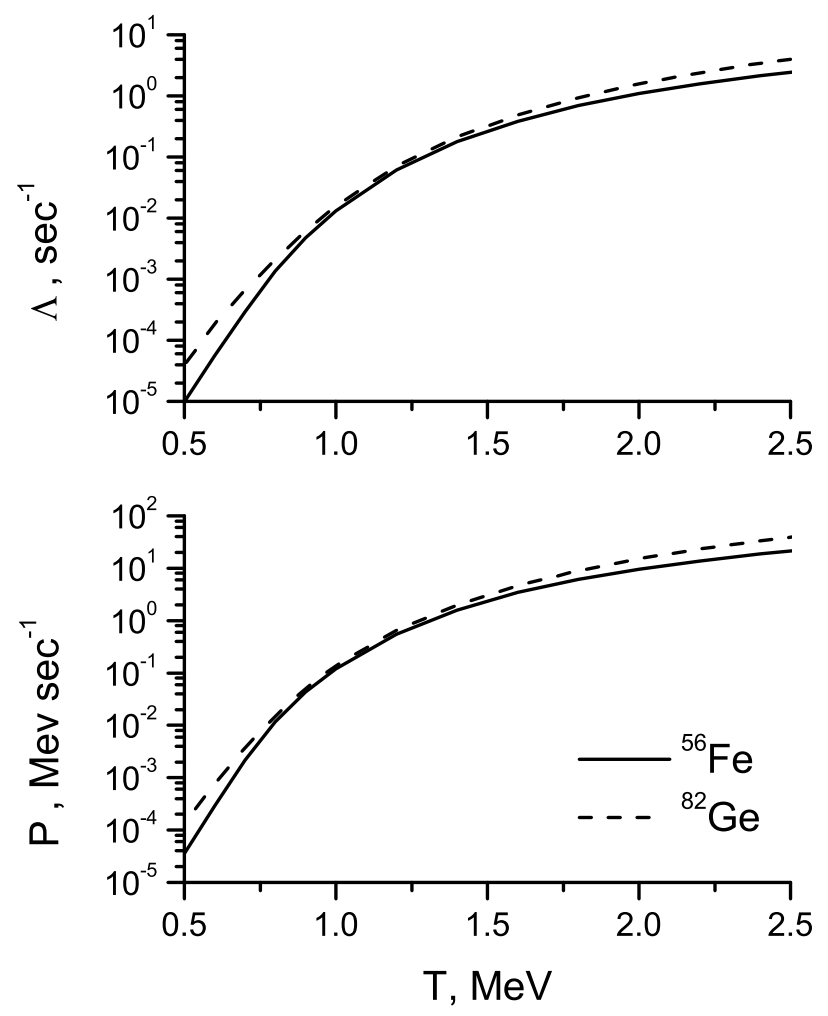

Figure 3. The total decay rate $\Lambda$ (upper panel) and the energy emission rate $P$ (lower panel) for ${ }^{56} \mathrm{Fe}$ and ${ }^{82} \mathrm{Ge}$ as functions of $T$.

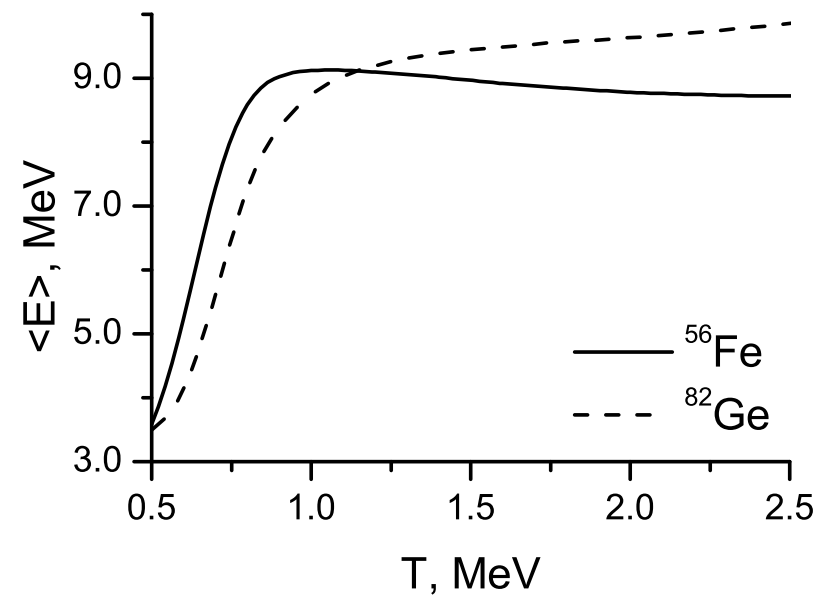

Figure 4. The mean energy $\langle E\rangle$ of $\nu \bar{\nu}$ pairs emitted from ${ }^{56} \mathrm{Fe}$ and ${ }^{82} \mathrm{Ge}$ as a function of temperature $T$. 